\title{
Der Status von Flüchtlingen in zwei deutschsprachigen Romanen aus der Perspektive des raumtheoretischen Ansatzes von Bernhard Waldenfels
}

Schlüsselwörter: Raumtheorie, Bernhard Waldenfels, Zugehörigkeit, Status von Flüchtenden, deutsche Gegenwartsliteratur

DOI: 10.4312/ars.13.2.89-101

Die Romane Wenn gefährliche Hunde lachen (2011) von Maxi Obexer und Havarie (2015) von Merle Kröger erzählen Fluchtgeschichten, wobei beide Texte auf die jüngsten Flüchtlingswellen vom afrikanischen Kontinent eingehen. Obexers Roman handelt von Helen, einer jungen Nigerianerin, die sich auf der Flucht nach Europa befindet und nach einer langen Odyssee schließlich nach Deutschland kommt. In Krögers Roman treten mehrere Figuren auf, deren Geschichten parallel erzählt werden, doch den Mittelpunkt der Handlung bildet das Schicksal einiger afrikanischer Flüchtlinge, die auf einem Schlauchboot im Mittelmeer treiben und schließlich die spanische Küste erreichen. In beiden Texten spielen Orte und Räume eine wichtige Rolle, worauf bereits Inhaltsverzeichnis bzw. Kapitelüberschriften der Romane hinweisen. Es fällt außerdem auf, dass beide Texte eine Differenzierung in topologischer Hinsicht vornehmen, nämlich eine Unterscheidung von Raum und Ort und damit verbunden eine Differenzierung des jeweiligen Subjektstatus. Die Flüchtlingsfiguren erfahren eine extreme Raumschrumpfung und eine Reduzierung auf einen Ort, was auf ihren Status als Subjekt zurückwirkt, wogegen bei den anderen Figuren, die keine Flüchtenden sind, eine ausgewogene Spannung zwischen Ortsfixierung und Raumausdehnung festzustellen ist. Nach Bernhard Waldenfels (vgl. Waldenfels, 2009) sind Menschen in der Regel an einem bestimmten Ort und gleichzeitig auch Teil eines auch diesen Ort umfassenden Raumes, zwischen beiden besteht also ein ausgewogenes Verhältnis. Dieses scheint jedoch in den beiden Romanen aus dem Gleichgewicht geraten zu sein, was im Folgenden anhand einer raumtheoretischen Analyse gezeigt werden soll. Dabei beziehe ich mich auf die raumtheoretischen Überlegungen von Waldenfels, die ich für die Analyse der beiden Romane operationalisieren möchte. Zunächst soll nun Waldenfels' Ansatz kurz vorgestellt werden, der danach auf die Lektüre der beiden Romane appliziert werden soll. ${ }^{1}$

1 Finanzielle Unterstützung durch die Slowenische Forschungsagentur (Forschungsgruppe Nr. P60265). 


\section{Raumtheoretische Ansätze bei Bernhard Waldenfels}

Den Ausgangspunkt der raumtheoretischen Überlegungen von Waldenfels bildet das Subjekt, "das leibliche Ich, das sich durch seine eigenen Bewegungen eine Welt erschließt." (Waldenfels, 2009, 19) Dabei geht das Subjekt von einem Hier und Jetzt aus, konstituiert sich also als "Hier-Jetzt-Ich-System" (ebd., 20), das zwischen Nahund Fernräumen und Innen- und Außenräumen differenziert. Das Subjekt ist also an einem Ort und bewegt sich gleichzeitig in einem Raum. Dabei ist der Ort mit dem jeweiligen Selbst verbunden und wirkt auf dessen Selbstverständnis zurück, denn jedes Individuum hat "seinen eigenen Ort, ohne den das Individuum kein Individuum wäre, und seinen angestammten, zugehörigen Ort, ohne den es kein besonders geartetes Individuum wäre." (Ebd., 119) Von diesem Ort abzugrenzen wäre ein Raum, "in den wir uns einordnen" (ebd., 12) und der die Orte umgibt, an denen sich Subjekte befinden und zu denen sie sich zugehörig fühlen, denn "ein Leben und Wohnen im Raum läßt sich nicht denken ohne eine innere Zugehörigkeit der Bewohner zu dem Ort, an dem sie sich aufhalten." (Ebd., 65) Dabei kann die Zugehörigkeit stärker oder schwächer sein, je nachdem, wie sehr das Ortsgefühl und das Raumwissen zusammenfallen. Ersteres bezeichnet das leibliche Gefühl, sich an einem bestimmten Ort zu befinden, letzteres das Wissen vom Raum, also das Wissen darüber, wie der Raum funktioniert und welche Ordnung ihn bestimmt. Das Gefühl der Fremdheit ist beispielsweise Resultat einer Abspaltung von Ortsgefühl und Raumwissen, d.h. das Gefühl, an einem Ort zu sein deckt sich nicht mit dem Wissen von dem Raum, in dem sich dieser Ort befindet.

In der Regel besteht eine ausgewogene Spannung zwischen der "Zentrierung am Ort und der Verteilung im Raum." (Ebd., 47) Gerät dieses Verhältnis aus dem Gleichgewicht, "droht sich das Hier zu verflüchtigen, der Raum sich zu verfestigen" (ebd.), was sich mit den Bezeichnungen "Ort ohne Raum und Raum ohne Ort" (ebd., Hervorhebung im Original) benennen lässt. Letzteres bezeichnet einen Raum, in dem es keine singulären Kennzeichnungen gibt, kein individuelles oder situatives Hier und Jetzt, wodurch die Existenz eines Ich im Sinne eines Individuums fragwürdig wird. Orte ohne Räume dagegen bezeichnen eine Reduzierung auf ein Hier und Jetzt, wobei der Raum als Faktor der Zugehörigkeit abhandenkommt, was letztendlich ebenso zu einer Abwesenheit eines Ich führt, da eine Verortung oder Einordnung in einen Raum der Zugehörigkeit nicht möglich ist.

Im Gegensatz zum Ort, der situativ und singulär ist und in gewisser Weise von einem Individuum besetzt wird, zeichnet den Raum eine Vielfalt aus, die einerseits durch seine Beschaffenheit als geschlossener, offener, dynamischer, statischer, ausgedehnter oder geschrumpfter Raum entsteht, andererseits aber auch durch 
die Vielzahl von Orten, die er umfasst. Insofern gibt es nicht den Raum und die Raumordnung, in den bzw. in die sich Subjekte einordnen, und auch keinen Raum, in dem alles seinen angestammten Platz hätte, wie das beispielsweise für den griechischen Kosmos gilt. "Den Raum gibt es nur in der Vielfalt von Raumweisen und in der raumübergreifenden Verkettung oder Verschmelzung verschiedenartiger Räume." (Ebd., 66) Dieses Verständnis vom Raum lässt die vermeintliche Homogenität des Raumes obsolet werden und unterstützt dagegen die Annahme, dass der Raum von der jeweiligen Erfahrung und dem jeweiligen Subjekt der Erfahrung abhängt.

Es soll nun versucht werden, mit Hilfe des raumtheoretischen Ansatzes von Waldenfels einige Aspekte der beiden Romane näher zu untersuchen. Für beide Romane gilt, dass die Flüchtenden eine extreme Raumschrumpfung und Ortfixierung erfahren. Insofern könnte behauptet werden, dass der genuine Ort von Flüchtenden ein "Ort ohne Raum" und "Raum ohne Ort" ist. In dem Roman Havarie fällt zudem auf, dass sich die Figuren in einem gemeinsamen Raum bewegen (das Mittelmeer), sich teilweise auch denselben Ort teilen (die unterschiedlichen Schiffe), sich aber gleichzeitig in unterschiedlichen Räumen befinden. Für sie gilt, was Waldenfels Ortsverschiebung nennt. Das bedeutet, dass jemand "zugleich hier und anderswo" (ebd., 124) ist, woraus folgt, dass jemand "zugleich er oder sie selbst und ein Anderer, eine Andere” (ebd.) ist. Für die Flüchtenden, die eine Reduzierung auf das Hier und Jetzt erleben und damit eine Raumschrumpfung, gilt das nicht mehr. Ihr Status, der sich aus der Doppelfigur von Ort ohne Raum und Raum ohne Ort ergibt, lässt sich mit Agamben als "nacktes Leben" (vgl. Agamben, 2002) bezeichnen, und was Agamben über die Insassen der Konzentrationslager schreibt, trifft auch für die in den Texten auftretenden Flüchtlingsfiguren zu: "Genau darum, weil sie aller Rechte und aller Erwartungen, die wir gewöhnlich mit der menschlichen Existenz verbinden, beraubt und dennoch biologisch noch am Leben sind, halten sie sich in einer Grenzzone zwischen Leben und Tod, zwischen Innen und Außen auf, wo sie nichts weiter mehr waren als nacktes Leben." (Agamben, 2002, 168)

\section{Raumtheoretische Aspekte im Roman Wenn gefährliche Hunde lachen}

Der Roman erzählt die Geschichte von Helen, die durch die Sahara über Marokko nach Deutschland flüchtet. Nach dem gefährlichen Weg durch die Wüste, in der sie immer wieder Schikanen seitens der Schlepper und der Grenzpolizei ausgesetzt ist, gelangt sie in das marokkanische Tanger. Dort haust sie gemeinsam mit anderen Flüchtenden in einem Wald und wartet auf die Überfahrt nach Europa. Begleitet wird sie von ihrem Freund Benjamin, einem jungen Eritreer, der sich jedoch als ambivalente 
Figur erweist, da nicht ganz klar ist, ob er nicht, da er Helen auf der Flucht immer wieder zu sexuellen Handlungen mit den Grenzpolizisten nötigt, ebenfalls als Schlepper fungiert. In Tanger zwingt er sie zur Prostitution. Als Helen vor ihm flieht, wird sie von einem Marokkaner aufgegriffen, der sie unter falschen Versprechungen in ein Haus bringt, wo sie sich waschen und ausruhen kann. Allerdings erweist sich der Mann als Zuhälter und das Haus als Bordell, in das Helen eingesperrt wird. Nach einiger Zeit gelingt es ihr, den Zuhälter davon zu überzeugen, sie gehen zu lassen, und gemeinsam mit anderen Flüchtlingen gelangt sie auf einem Schlauchboot nach Spanien, wo sie in ein Flüchtlingscamp gebracht wird. Helen ist schwanger, allerdings wird das Kind ohne ihr Wissen mit Tabletten abgetrieben. Schließlich gelingt ihr die Flucht aus dem Lager und, versteckt in einem Lastwagen, kommt sie nach Deutschland. Dort bittet sie auf einer Polizeistation um Asyl und wird von den Beamten mit dem Zug quer durch Deutschland in ein Asylantenheim geschickt, wo sie auf ihre Anhörung wartet. Helen erkrankt an einer starken Depression und lebt mehrere Monate in einem Zustand der völligen Abwesenheit, weswegen sie in die Psychiatrie eingeliefert wird.

Betrachtet man Helens Fluchtgeschichte topologisch, so ergibt sich eine klare Differenzierung in Raum und Ort. Die im Roman auftauchenden Räume - Afrika, Europa, die Wüste, das Mittelmeer - fungieren hierbei als Räume, die Helen zwar durchschreitet, zu denen sie jedoch nicht gehört, wobei es jedoch zwischen diesen Räumen zu differenzieren gilt. Obwohl ihre afrikanische Heimat sie als Flüchtende ebenso ausschließt wie Europa, das sie nicht aufnehmen möchte, fungieren beide Räume in gewisser Hinsicht immer noch als Räume der Zugehörigkeit, denn sie werden zu imaginären Räumen, zu imaginierten Fluchtorten, in denen sich Helen bewegt, um die reale Flucht überhaupt aushalten zu können. "Denk dich woandershin, du bist einfach woanders. [...] hier sind alle europäischen Städte vertreten, zumindest in ihrer Phantasie, so wie sich fast alle afrikanischen Länder hier finden lassen.” (Obexer, 2011, 11-12) Sind Europa und Afrika zumindest in der Phantasie noch Räume der Zugehörigkeit, in denen ein Leben erinnert werden kann und vorstellbar ist, erscheinen die Wüste und das Mittelmeer als eigentliche Grenzräume, die Helen durchschreiten muss. Zu diesen Räumen gibt es keine Zugehörigkeit, sie gleichen “Totenfeldern” (ebd., 38) oder der "Hölle” (ebd., 14), Räumen, die ihre eigene "'Rechts'Ordnung” (Steidl 2017, 313) entwickeln, in denen eigene Regeln herrschen, und die vor allem durch Gewalt gekennzeichnet sind. Obwohl sich Grenzräume in der Regel dadurch auszeichnen, dass in ihnen - ähnlich wie in Schwellenräumen, Limitrophien oder dem third space (vgl. Borgards, 2012, 10) -, durch das Aufeinandertreffen von Ordnungen neue Ordnungen entstehen und die gemeinhin als Räume des Aushandelns verstanden werden, gilt genau das für die Grenzräume, in denen sich Helen bewegt, nicht mehr. Diese unterscheiden sich dadurch, dass der Mensch in ihnen auf das 
"nackte Leben" reduziert wird und andererseits bieten sie auch keine Möglichkeit des Aushandelns mehr. In diesen Räumen herrscht ein permanenter Ausnahmezustand, in dem prinzipiell alles möglich ist, und in ihnen gibt es weder ein subjektives Recht noch einen rechtlichen Schutz.

Weder die Wüste noch das Meer bieten eine Möglichkeit der Verortung, d. h., es gibt keine Möglichkeit, sich als "Hier-Jetzt-Ich-System" (Waldenfels, 2009, 43) in diesen Räumen zu konstituieren, wovon nicht nur Metaphern wie “Totenfelder” und "Massengräber" (Obexer, 2011, 111) zeugen, sondern auch die Leere, die Helen in einem ihrer Briefe beschreibt: "Liebe Eltern, in der Sahara unterwegs zu sein ist wie im Meer sein. [...] Du siehst über Tausende von Kilometern nichts, nur Dünen in jeder Himmelsrichtung. [...] Man kann oft über mehrere Stunden nicht sehen, dass sich etwas bewegt, außer dem Sand, der vom Wind bewegt wird. Keine Tiere, keine Bäume, keine Menschen." (Ebd., 27-28) Sowohl diese Grenzräume als auch Afrika und Europa, die zwar eine - wenn auch lediglich imaginäre Zugehörigkeit ermöglichen, sind Räume ohne Orte, Räume also, die sich verfestigen und dem Subjekt kein individuelles und situatives Hier und Jetzt bieten. Der Raum ist übermächtig und verschluckt sozusagen das Ich, bestimmt dessen Sein. In diesen Räumen gelangt Helen immer wieder an Orte, die eine Ausdehnung oder Bewegung des Ich entweder ganz oder zumindest teilweise einschränken. $\mathrm{Zu}$ unterscheiden sind hierbei dynamische Orte, in die sie eingeschlossen ist (Wagen, Kleinlaster, Lastwagen, Zug) und statische Orte, die das Subjekt festsetzen (Gefängnis, Stall, Container, Sandkuhle, Keller, Bordell, Zimmer, Zelt, Lager, Krankenzimmer, Erstaufnahmelager, Asylantenheim, Psychiatrie). In beiden Fällen handelt es sich um Orte ohne Raum, an denen Helen eine extreme Reduzierung ihres Selbst erfährt. Da Orte durch "die Verbindung des Ortes mit einem Selbst" (Waldenfels, 2009, 32) gekennzeichnet sind, ergibt sich die Frage nach dem Sein, dem Status dieses Selbst, vor allem auch deswegen, weil es sich um negativ konnotierte, klaustrophobische Orte handelt: das Erdloch in Tanger, in dem Helen auf die Überfahrt nach Europa wartet, das Bordell, die Psychiatrie. An diesen Orten wird der Mensch auf sein körperliches Sein reduziert, wie "leblos" (Obexer, 2011, 9) wartet er darauf, dass die Zeit vergeht, die es aber an diesen Orten gar nicht zu geben scheint: "Diese Zeit hier gibt es nicht, die zählt nicht." (Ebd., 11) Der Flüchtende gehört "nicht der menschlichen Gattung an" (ebd., 32), sondern wird zu einer "stumpfen Sorte Tier, das hinnimmt, was für normale Menschen nicht hinnehmbar ist.” (Ebd.) Helen und die anderen Flüchtlinge sind so gesehen keine Ortlosen, sondern Raumlose, die durch den Verlust ihres Raumes auf das Sein an Orten reduziert werden, mit denen sie identisch werden: "Keiner sagt uns etwas, keiner macht sich die Mühe mit uns zu sprechen, die Art, wie wir zusammengepfercht gehalten werden, wie Geflügel, gleichgültig, sinnlos, zeitlos [...]." (Ebd., 116) Diesen Orten ist die paradoxe Verortung 
außerhalb und innerhalb ebendieser Räume eigen, denn die Flüchtenden sind aus den als verbindlich geltenden Rechtsräumen ausgeschlossen, befinden sich aber nicht in irgendeinem Außerhalb, sondern in einem von diesen Rechtsräumen als solches definiertem Außerhalb. Der Flüchtende ist an diesen Orten tatsächlich auf die Existenz des Agambschen homo sacer (Agamben, 2002, 168) reduziert, der in einem Maße entmenschlicht wird - und zwar in dem Sinne, als er seiner grundlegenden politischen Rechte beraubt wird -, dass er getötet werden kann, ohne dass diese Tötung rechtliche Konsequenzen nach sich ziehen würde. Insofern befinden sich diese Orte innerhalb eines Systems, das diese Behandlung zulässt, und gleichzeitig außerhalb des Systems, da hier nicht dieselben Regeln herrschen wie im gesamten System.

Die Differenzierung in Ort und Raum, die Waldenfels vorschlägt, macht eine differenziertere Beschreibung möglich und rückt darüber hinaus die Frage nach dem Status des Selbst, das sich an solchen Orten befindet, stärker in den Fokus, denn Orte sind ja immer auch mit dem dort seienden Selbst verbunden und wirken darauf zurück. Die Orte und Räume unterscheiden sich einerseits aufgrund ihrer Beschaffenheit und andererseits nach der Art, wie das Subjekt sich selbst dort erfährt, was nun anhand von einigen Beispielen veranschaulicht werden soll.

Die Wüste als offener und ausgedehnter Raum, als "Öde, die nicht aufhört, die kein Ende nimmt" (Obexer, 2011, 29), erfährt Helen als feindlichen Raum, dem sie ausgeliefert ist und in dem sie verloren geht, als Raum ohne Ort also, der das Subjekt verschluckt. Gleichzeitig erfährt sie dort auch die totale Schrumpfung des Raums auf Orte, die das Subjekt bis zur Auflösung reduzieren. Hierzu gehören beispielsweise die Sandkuhlen, in die sich die Flüchtlinge eingraben. Jeder hat eine "eigene Sandkuhle" (ebd., 23) und unter "dem Sand, den der Wind auf ihnen ablegt, verschwinden in kurzer Zeit die bunten Farben ihrer Kleidung; sie tragen nun alle dieselbe hellbraune Farbe der Wüste." (Ebd.) Hier gibt es kein individuelles Sein mehr - weder in der Ausdehnung des Raumes, noch in der Fixierung auf einen Ort, denn beide Erfahrungen führen zu einer "Schwächung des leiblichen Hier, die sich seiner Aufhebung mehr oder weniger annähert" (Waldenfels, 2009, 96), zu einem Verlust des Selbst also, der dazu führt, dass irgendwann "nichts mehr von dir übrig bleibt." (Obexer, 2011, 18) Trotzdem sie hier eine extreme Reduzierung erfährt, birgt die Offenheit des Raumes doch eine Dynamik in sich, die sich in Helens Hoffnungen zeigt und auch in der fast poetischen Beschreibung der Wüste als "Seereise auf dem Land" (ebd., 27), denn manchmal "schimmert die Luft so, dass man meinen könnte, man befände sich mitten auf einem von Licht gleisendem See” (ebd.), der ständig seine Form ändert. Diese Möglichkeit einer Veränderung kommt in den geschrumpften Räumen, den “Orten ohne Raum”, an denen Helen sich befindet, völlig abhanden, wovon Helens Eindrücke aus dem Bordell zeugen: "Sie sieht, wie ihre Augen sind, wenn sie [die Mädchen, Anm. A. L.] in dieses 
Haus kommen, und sie sieht ihre Augen nach zehn Tagen. Es ist keine Bewegung mehr darin zu erkennen, sie leben nicht mehr, sie sterben nicht, denn auch dazu musst du noch den Hauch eines Lebens besitzen." (Ebd., 98) An diesen Orten vollzieht sich eine Reduzierung des Menschen auf sein nacktes Leben: Er ist nur noch Körper, wovon auch die Psychiatrie als absolut geschlossener Ort zeugt: "Mein Kopf jedenfalls war nicht mehr hier und nicht bei mir. Ich habe keine Ahnung, wo er stattdessen war. Nur der Körper war noch hier. Der Körper aber rannte kopflos umher, nervös wie er war, verloren wie er war ohne Kopf." (Ebd., 159) Helens Fluchtgeschichte ist eine Bewegung durch schrumpfende Räume und endet schließlich an einem absolut geschlossenen Ort. Sie zeichnet damit auch die Entmenschlichung des Flüchtenden nach, der durch den Verlust jeglichen Raums auf die Existenz als Körper reduziert wird.

\section{Raumtheoretische Aspekte im Roman Havarie}

Auch in dem Roman Havarie spielen Räume und Orte eine ausschlaggebende Rolle, worauf nicht nur die Kapitelüberschriften hinweisen, die exakte Ortsbeschreibungen sind, sondern auch die Fotografien unterschiedlicher Orte im Anhang. Im Unterschied zu Obexers Roman, in dem eine Hauptfigur im Mittelpunkt steht, treten in Havarie mehrere Figuren mit unterschiedlichen Geschichten auf, die jedoch topologisch miteinander verbunden sind. Die Handlung umfasst drei Tage und entfaltet sich an verschiedenen Orten, die das Mittelmeer als Handlungsraum miteinander verbindet. Bei den Orten handelt es sich um unterschiedliche Schiffe bzw. Boote, die nicht nur durch den gemeinsamen Raum, das Meer, miteinander verbunden sind, sondern auch durch das Schicksal der Flüchtlinge, auf dem der Fokus des Romans liegt. Diese befinden sich auf einem Gummiboot auf dem Mittelmeer und werden in der Nacht von dem Luxusdampfer Spirit of Europe gesichtet. Anstatt die Flüchtlinge an Bord zu nehmen, versorgt sie die Besatzung mit Decken und Nahrungsmitteln und schickt sie zurück aufs Meer. Außerdem wird Marwan, ein afrikanischer Flüchtling, der sich illegal auf dem Luxusdampfer befindet, auf das Gummiboot gebracht, das am nächsten Tag die spanische Küste erreicht, wo Marwan stirbt.

Topologisch betrachtet gibt es auch in diesem Text eine deutliche Differenzierung in Raum und Ort. Gemeinsamer Handlungsraum ist das Mittelmeer, auf bzw. an dem sich die unterschiedlichen Handlungsorte befinden: der Luxusdampfer, das Gummiboot, ein Frachter, ein Fischerboot, ein Rettungsboot sowie die Häfen von Oran und Cartagena und das spanische Portmán. Jedem der Orte sind bestimmte Figuren zugeordnet. Einerseits sind die Figuren durch die Orte bestimmt und dadurch mit der Handlung verbunden, andererseits breiten sie sich aber in individuelle Räume aus, die nicht direkt mit der Handlung in Verbindung stehen, diese aber in einem 
gewissen Sinne reflektieren. Es handelt sich dabei um Orte, die ihrerseits durch Flucht und Krieg gekennzeichnet sind, wie zum Beispiel das Flüchtlingsschiff Gustloff, der Bürgerkrieg in Belfast oder Migrationserfahrungen an unterschiedlichen Orten. So entstehen unterschiedliche Zeit- und Raumebenen: das Hier und Jetzt der Handlung und die jeweiligen Vergangenheiten, sowie die damit verbundenen Orte, die im Text nicht Handlungsorte sondern Erinnerungsorte sind, worauf auch die Fotografien im Anhang hinweisen. Jede Figur ist also an einem bestimmten Ort lokalisiert, erschließt sich aber gleichzeitig in Gedanken einen anderen Raum, zu dem sie sich zugehörig fühlt. Der Ort, an dem sich die Figuren befinden, ist situativ bestimmt (Kreuzfahrt, Fischerei, Rettungsdienst usw.) und bestimmt teilweise auch das Sein des Individuums; andererseits sind die Figuren durch den Raum bestimmt, in den sie sich ausdehnen und zu dem sie sich zugehörig fühlen. Das gilt für alle Figuren gleichermaßen, außer für den Flüchtling Marwan, dessen Raum zusehend schrumpft.

So entsteht eine Dichotomie von Figuren hinsichtlich der erfahrenen Raumausdehnung und -schrumpfung. Für diejenigen, die sich in den Raum ausdehnen können gilt, was Waldenfels Ortsverschiebung nennt, nämlich ein gleichzeitiges Sein im Hier und Jetzt und in einem Anderswo. So entsteht ein Kaleidoskop von Geschichten, andererseits aber auch ein überindividueller Raum, in dem sich die je individuellen Räume ineinander verketten. Alle Figuren erinnern nämlich Erlebnisse, die sie mit den Flüchtenden auf dem Meer in Verbindung bringen: Flucht, Gewalt, Krieg, Diskriminierung, Fremdheit. Insofern sind sie tatsächlich sie selbst und jemand anderes, was Ortverschiebung ja impliziert. Eine Ausnahme bildet Marwan. Genau wie Helen erfährt er eine zunehmend radikaler werdende Raumschrumpfung. Er arbeitet in der Wäscherei des Dampfers und beschreibt das wie folgt: "Wir sind Verdammte auf diesem Totenschiff. Wir sind längst tot. Wir brauchen nichts: Kein Tageslicht in der Wäscherei. Kein Tageslicht in der Kabine. Kein Tageslicht in der Crewkantine. Eine verstohlene Zigarette draußen vor dem Trainingsraum. Kurzer Blick zum Himmel. Schönstes Urlaubswetter. Nicht für uns. Tote brauchen keinen Urlaub.” (Kröger, 2015, 24) Zu Beginn des Romans befindet sich auch Marwan gedanklich noch in anderen Räumen: Er erinnert sich an Syrien, an seine Arbeit als Arzt, an seine Eltern. Die Möglichkeiten einer gedanklichen Flucht werden jedoch immer geringer und bleiben schließlich ganz aus. Nachdem Marwan erkrankt, wird er, da man ihn auf dem Luxusschiff nicht mehr duldet, auf das Schlauchboot gebracht, das in der Nacht gesichtet wird. Das Boot ist allerdings kein Ort ohne Raum, denn es befindet sich ja - und zwar als dynamischer Ort - mitten im Raum, dem Mittelmeer, das alle anderen Räume miteinander verbindet. Die Dynamik des Ortes zeigt sich beispielsweise an Karim, der das Boot auch lenkt. Während der Überfahrt schweift er in Gedanken immer wieder in jene Räume ab, zu denen er eigentlich gehört: Algerien, 
seine Familie, seine Verlobte. Diese Räume der Zugehörigkeit sichern sein Überleben, geben ihm Hoffnung und bilden einen Raum, in den hinein er sich ausdehnen kann. Das Mittelmeer, das für alle Figuren ein Raum ist, in dem sie sich an unterschiedlichen Orten lokalisieren können, wird allein für Marwan zum Raum ohne Ort, in dem er verloren geht. Immer wieder fragt er “wo bin ich?” (ebd., 49, 131), eine Frage, die auf die Abwesenheit eines Ortsgefühls und eines Raumwissens hinweist und damit auf die völlige Ort- und Raumlosigkeit. Das Abhandenkommen des Raums zeigt sich außerdem in seinen Erinnerungen, die sich in einem Maße ineinanderschieben, dass sich die raumzeitlichen Grenzen zwischen ihnen auflösen und dem Leser lediglich Bruchstücke von Marwans Geschichte vermitteln. Gemeinsam mit den anderen Flüchtenden auf dem Schlauchboot erreicht Marwan einen Strand im spanischen Portmán, wo er stirbt. Dieser Strand ist ein Ort ohne Raum und wird folgendermaßen von Marwan erfahren: "Sand. Salziges Wasser. Sein Kopf schmerzt unerträglich. Es tobt ein Gewitter in seinem Kopf. Er möchte sich hinlegen. Im Sand versinken. Er versinkt nicht. Starke Arme greifen ihn links und rechts und tragen ihn. Seine Beine sind butterweich. Trockener Sand. Unter ihm schwarzer Sand. Über ihm schwarzer Himmel. Die Sonne, die Farben haben Marwan verlassen. [...] Hallo? Ist hier jemand? Ich kann nichts sehen. Es ist dunkel. Mir ist kalt. Ich möchte aufstehen. Er tastet mit den Händen herum. Ich möchte nach Hause gehen.” (Ebd., 220-221) Marwan ist nur noch wahrnehmender Körper, der nicht mehr agieren kann, also kein Subjekt im eigentlichen Sinne. Der Strand, an dem er schließlich stirbt, erinnert an jenen Ort, den Agamben als "extratemporale und extraterritoriale Schwelle" (Agamben, 2002, 168) bezeichnet, "wo der menschliche Körper von seinem normalen politischen Status losgelöst ist und so in einem Ausnahmezustand den extremsten Wechselfällen überlassen wird." (Ebd.) Marwan ist tatsächlich jener homo sacer, der "getötet werden kann, ohne daß ein Mord begangen wird." (Ebd.) Der Flüchtling Karim, der das Gummiboot lenkt, nimmt schließlich Marwans Pass an sich und damit seine Identität. Von Marwan bleibt nichts mehr übrig außer seinem Körper. Er ist ein "Namenloser" (Kröger, 2015, 26), ohne Identität.

\section{Schlussbetrachtungen}

Da Fluchtgeschichten und Flüchtende eine "besondere Beziehung zum Raum" (Hardtke et al., 2017, 18) haben, ist eine raumanalytische Lektüre von Fluchtgeschichten sicherlich aufschlussreich, um die "zeitlichen und räumlichen Transformationen zu beschreiben, die die Existenz Geflüchteter ausmachen.” (Ebd., 12) Allerdings unterliegen nicht nur die Flüchtenden diesen Transformationen, sondern auch der Raum, den sie durchschreiten, denn wir "bewegen uns von Ort zu Ort, aber auf Wegen, die sich in den Raum einzeichnen und auf den Bewegungsablauf zurückwirken.” (Waldenfels, 2009, 
50) Insofern wäre auch zu fragen, was die Flucht mit den Räumen macht, wie sich diese aufgrund der Erfahrung mit Flüchtenden verändern, was im Hinblick auf die steigende Fremdenfeindlichkeit in Europa und den damit verbundenen Populismus aufschlussreich sein könnte. Mit Hilfe von raumtheoretischen Analysen lassen sich außerdem jene Orte und Räume näher beschreiben, an bzw. in denen Flüchtende sich aufhalten und die auf ihren Status als Mensch zurückwirken, was im vorliegenden Aufsatz ansatzweise versucht wurde. Die Begriffe "Raum ohne Ort" und "Ort ohne Raum" bezeichnen meines Erachtens den spezifischen Status von Flüchtenden, der in einer grundlegenden Rechtslosigkeit und Entmenschlichung und in einer extremen Raumschrumpfung liegt, die zu einer Aufhebung jeglicher Zugehörigkeit führt und ihnen den Status eines Subjekts abspricht. Gerade die beiden konstitutiven Aspekte Zugehörigkeit und Reduzierung des Subjekts auf sein nacktes Leben grenzen diese beiden Begriffe von Begriffen wie Schwellenräumen, Limitrophien oder dem third space ab, die in der Regel Räume des Aushandelns sind, in denen fremde und eigene Ordnungen aufeinandertreffen und neue Ordnungen entstehen können. Verglichen mit den Orten und Räumen, an bzw. in denen sich Helen und Marwan aufhalten, erscheinen solche Aushandlungsräume als bloße Euphemismen.

\section{Bibliographie}

Agamben, G., Homo sacer. Die Souveränität der Macht und das nackte Leben, Frankfurt a. M. 2002.

Borgards, R., Liminale Anthropologien. Skizze eines Forschungsfeldes, in: Liminale Anthropologien (Hg. Achilles, J., Borgards, R., Burrichter, B.), Würzburg 2012, S. 9-13.

Hardtke, T. et al., Niemandsbuchten und Schutzbefohlene. Flucht-Raume und Fluchtlingsfiguren in der deutschsprachigen Gegenwartsliteratur, in: Niemandsbuchten und Schutzbefohlene. Flucht-Raume und Fluchtlingsfiguren in der deutschsprachigen Gegenwartsliteratur (Hrsg. Hardtke, T. et al.), Gottingen 2017, S, 9-20.

Kröger, M., Havarie, Hamburg 2015.

Obexer, M., Wenn gefährliche Hunde lachen, Wien, Bozen 2011.

Steidl, S., Der Flüchtling als Grenzgestalter? Zur Dialektik des Grenzverletzers in Abbas Khiders Debütroman Der falsche Inder, in: Niemandsbuchten und Schutzbefohlene. Flucht-Räume und Flüchtlingsfiguren in der deutschsprachigen Gegenwartsliteratur (Hrsg. Hardtke, T. et al.), Göttingen 2017, S. 305-320.

Waldenfels, B., Ortsverschiebungen, Zeitverschiebungen, Frankfurt a. M. 2009. 


\section{Der Status von Flüchtlingen in zwei deutschsprachigen Romanen aus der Perspektive des raumtheoretischen Ansatzes von Bernhard Waldenfels}

Schlüsselwörter: Raumtheorie, Bernhard Waldenfels, Zugehörigkeit, Status von Flüchtenden, deutsche Gegenwartsliteratur

Bernhard Waldenfels unterscheidet zwischen einem Raum, in den sich jemand einordnet und zu dem er gehört, und einem Ort, an dem sich jemand befindet. Das Subjekt ist also an einem Ort und bewegt sich gleichzeitig in einem Raum. In der Regel besteht eine ausgewogene Spannung zwischen dem Ort und der Verteilung im Raum. Gerät dieses Verhältnis aus dem Gleichgewicht, entstehen Orte ohne Raum und Räume ohne Orte. Letzteres bezeichnet einen Raum, in dem es keine singulären Kennzeichnungen gibt, kein individuelles oder situatives Hier und Jetzt, wodurch die Existenz eines Ich im Sinne eines Individuums fragwürdig wird. Orte ohne Räume dagegen bezeichnen eine Reduzierung auf ein Hier und Jetzt, wobei der Raum als Faktor der Zugehörigkeit abhandenkommt, was letztendlich ebenso zu einer Abwesenheit eines Ich führt, da eine Verortung oder Einordnung in einen Raum der Zugehörigkeit nicht möglich ist. Der Aufsatz ist der Versuch, diesen raumtheoretischen Ansatz für die Analyse literarischer Texte zu operationalisieren, in deren Mittelpunkt Flüchtende stehen. 
Andrea Leskovec

\section{Status beguncev $\mathbf{v}$ dveh sodobnih nemških romanih skozi prizmo Waldenfelsove teorije prostora}

Ključne besede: teorija prostora, Bernhard Waldenfels, pripadnost, status beguncev, sodobna nemška literatura

Bernhard Waldenfels razlikuje med prostorom, v katerega se posameznik uvrsti in h kateremu pripada, in krajem, na katerem se posameznik nahaja. Razmerje med tem, da se subjekt nahaja na določenem kraju in se hkrati razprostira $v$ določenem prostoru, je ponavadi uravnoteženo. Ko se to razmerje podira, nastanejo »kraji brez prostora in "prostori brez kraja«. Pri slednjih gre za prostore brez individualnega in specifičnega »tukaj« in »zdaj«, kar močno ogroža posameznika kot takšnega, »kraj brez prostora « pa pomeni redukcijo na določen »tukaj« in »zdaj«, ki posamezniku ne omogoča pripadnosti, kar privede do odsotnosti jaza, ker se posameznik ne more niti lokalizirati niti uvrstiti v določen prostor. Pričujoči prispevek je poskus analize dveh romanov s perspektive Waldenfelsove teorije prostora. 
Andrea Leskovec

\section{The Status of Refugees in Two German-Language Novels from the Perspective of the Space-Theoretical Approach of Bernhard Waldenfels}

Keywords: theory of space, Bernhard Waldenfels, affiliation, status of refugees, German contemporary literature

Bernhard Waldenfels distinguishes between a space which someone arranges and that he or she belongs to, and a place where someone is. So the subject is in one place and at the same time moves in a space. In general, there is a balanced tension between the location and distribution in space. If this relationship becomes unbalanced, places without space and spaces without places arise. The latter designates a space in which there are no singular identifications, no individual or situational here and now, whereby the existence of an ego in the sense of an individual becomes questionable. Places without spaces, on the other hand, signify a reduction to a here and now, where space is lost as a factor of affiliation, which ultimately leads to an absence of an ego, since it is not possible to locate or classify it in a space of belonging. The essay is an attempt to operationalise this spatial-theoretical approach to the analysis of literary texts, which focus on refugees. 